\title{
DLHub: Model and Data Serving for Science
}

\author{
Ryan Chard*, Zhuozhao $\mathrm{Li}^{\dagger}$, Kyle Chard ${ }^{\ddagger *}$, Logan Ward ${ }^{\dagger *}$, Yadu Babuji ${ }^{\ddagger}$, Anna Woodard ${ }^{\dagger}$, \\ Steven Tuecke ${ }^{\ddagger}$, Ben Blaiszik ${ }^{\ddagger *}$, Michael J. Franklin ${ }^{\dagger}$, and Ian Foster*†‡, \\ ${ }^{*}$ Data Science and Learning Division, Argonne National Laboratory, Argonne, IL, USA \\ ${ }^{\dagger}$ Department of Computer Science, University of Chicago, Chicago, IL, USA \\ ${ }^{\ddagger}$ Globus, University of Chicago, Chicago, IL, USA
}

\begin{abstract}
While the Machine Learning (ML) landscape is evolving rapidly, there has been a relative lag in the development of the "learning systems" needed to enable broad adoption. Furthermore, few such systems are designed to support the specialized requirements of scientific ML. Here we present the Data and Learning Hub for science (DLHub), a multi-tenant system that provides both model repository and serving capabilities with a focus on science applications. DLHub addresses two significant shortcomings in current systems. First, its selfservice model repository allows users to share, publish, verify, reproduce, and reuse models, and addresses concerns related to model reproducibility by packaging and distributing models and all constituent components. Second, it implements scalable and low-latency serving capabilities that can leverage parallel and distributed computing resources to democratize access to published models through a simple web interface. Unlike other model serving frameworks, DLHub can store and serve any Python 3compatible model or processing function, plus multiple-function pipelines. We show that relative to other model serving systems including TensorFlow Serving, SageMaker, and Clipper, DLHub provides greater capabilities, comparable performance without memoization and batching, and significantly better performance when the latter two techniques can be employed. We also describe early uses of DLHub for scientific applications.
\end{abstract}

Index Terms-Learning Systems, Model Serving, Machine Learning, DLHub

\section{INTRODUCTION}

Machine Learning (ML) is disrupting nearly every aspect of computing. Researchers now turn to ML methods to uncover patterns in vast data collections and to make decisions with little or no human input. As ML becomes increasingly pervasive, new systems are required to support the development, adoption, and application of ML. We refer to the broad class of systems designed to support ML as "learning systems." Learning systems need to support the entire ML lifecycle (see Fig. 1), including model development [1,2]; scalable training across potentially tens of thousands of cores and GPUs [3]; model publication and sharing [4]; and low latency and highthroughput inference [5]; all while encouraging best-practice software engineering when developing models [6].

While many systems focus on building and training ML models [3, 7, 8], there is a growing need for systems that support other stages of the ML lifecycle and in particular those important in science, such as publishing models used in the literature; low-latency serving of trained models for use in real-time applications; seamless retraining and redeployment of models as new data are available; and model discovery, reuse, and citation.

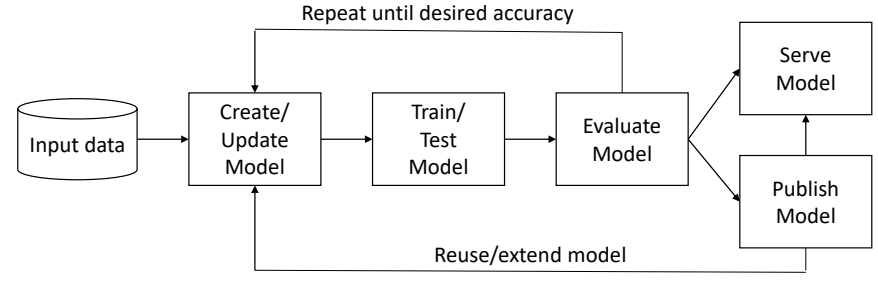

Fig. 1: ML lifecycle, adapted from Miao et al. [6]

The Data and Learning Hub for science (DLHub) is a learning system designed to address inefficiencies in two important phases of the ML lifecycle, namely the publication and serving of ML models plus associated data. DLHub provides a nexus for ML practitioners and model creators to publish and share models while capturing model provenance and providing credit to contributors. It includes a flexible, user-managed model catalog built upon a common metadata schema to enable discovery. DLHub also provides a low-latency and scalable model serving framework for executing inference and other tasks on arbitrary data, and for linking multiple data processing and model execution steps into end-to-end pipelines. This framework can execute containerized DLHub models efficiently on a variety of parallel and distributed computing resources for both low latency and high throughput.

Using DLHub, users can securely share and publish models and transformation codes, discover cutting-edge models published by the community, compose multi-step inference pipelines with pre-/post-processing stages, and perform inference at scale. Unlike other model repositories and serving systems, DLHub supports a wide range of model types including TensorFlow [8], Keras [9], and Scikit-learn [10]; integrates with Globus [11] to provide seamless authentication and high performance data access for training and inference; scales execution environments dynamically to provide scalable and low-latency inference; and supports workflows that link pre/post-processing steps and ML models.

In the following, we first motivate the need for science learning systems in $\$$ II and survey model repository and serving frameworks in III. We then describe the DLHub architecture and implementation in $\$ \mathrm{IV}$, explaining how it extends prior research efforts, for example by supporting both a wider variety of model types and the end-to-end workflows required for scientific uses of ML. In $\$ \mathrm{~V}$, we present experimental 
results which show that DLHub's scalable serving solution performs comparably with other systems, such as TensorFlow Serving [12] and SageMaker [3], and significantly better when memoization and batching can be employed. We show that without memoization, DLHub can serve requests to run models in less than 40ms and Python-based test functions in less than $20 \mathrm{~ms}$ on a Kubernetes cluster. Finally, we describe early uses of DLHub in $\S \mathrm{VI}$ and conclude in $\$ \mathrm{VII}$.

\section{Specialized ReQuirements of ScIENCE}

Increasingly sophisticated learning systems are being developed, in particular by the major cloud providers, to support commercial ML use cases. However, scientific use of ML has specialized requirements, including the following.

Publication, citation, and reuse: The scholarly process is built upon a common workflow of publication, peer review, and citation. Progress is dependent on being able to locate, verify, and build upon prior research, and careers are built upon publications and citation. As scholarly objects, ML models should be subject to similar publication, review, and citation models on which the scholarly process is built. Lacking standard methods for doing so, (a) many models associated with published literature are not available [13]; and (b) researchers adopt a range of ad hoc methods (from customized websites to GitHub) for sharing ML models [14-16].

Reproducibility: Concerns about reproducibility are having a profound effect on research [17]. While reproducibility initiatives have primarily focused on making data and experimental processes available to reproduce findings, there is a growing interest in making computational methods available as well [18,-20].

Unlike sharing software products, there is little guidance for sharing ML models and their artifacts (e.g., weights, hyper-parameters, and training/test sets). Without publishing these artifacts, it is almost impossible to verify or build upon published results. Thus, there is a growing need to develop standard ML model packages and metadata schema, and to provide rich model repositories and serving platforms that can be used to reproduce published results.

Research infrastructure: While industry and research share common requirements for scaling inference, the execution landscape differs. Researchers often want to use multiple (often heterogeneous) parallel and distributed computing resources to develop, optimize, train, and execute models. Examples include: laboratory computers, campus clusters, national cyberinfrastructure (e.g., XSEDE [21], Open Science Grid [22]), supercomputers, and clouds. Thus, we require learning systems that can support execution on different resources and enable migration between resources.

Scalability: Large-scale parallel and distributed computing environments enable ML models to be executed at unprecedented scale. Researchers require learning systems that simplify training and inference on enormous scientific datasets and that can be parallelized to exploit large computing resources.

Low latency: ML is increasingly being used in real-time scientific pipelines, for example to process and respond to events generated from sensor networks; classify and prioritize transient events from digital sky surveys for exploration; and to perform error detection on images obtained from X-ray light sources. There is a need in each case for low latency, near real-time ML inference for anomaly/error detection and for experiment steering purposes. As both the number of devices and data generation rates continue to grow, there is also a need to be able to execute many inference tasks in parallel, whether on centralized or "edge" computers.

Research ecosystem: Researchers rely upon a large and growing ecosystem of research-specific software and services: for example, Globus [11] to access and manage their data; community and institution-specific data sources (e.g., the Materials Data Facility [23] and Materials Project [24]) as input to their ML models; and research authentication and authorization models (e.g., using campus or ORCID identities).

Workflows: Scientific analyses often involve multiple steps, such as the staging of input data for pre-processing and normalization, extraction of pertinent features, execution of one or more ML models, application of uncertainty quantification methods, post-processing of outputs, and recording of provenance. There is a growing need to automate invocation of such sequences of steps into cohesive, shareable, and reusable workflows. It is important, furthermore, that such processing workflows can be adapted easily to meet the specific needs of a particular application.

\section{LEARNING SYSTEMS: A BRIEF SURVEY}

We define a learning system as "a system that supports any phase of the ML model lifecycle including the development, training, inference, sharing, publication, verification, and reuse of a ML model." To elucidate the current landscape, we survey a range of existing systems, focusing on those that provide model repository and serving capabilities. Model repositories catalog collections of models, maintaining metadata for the purpose of discovery, comparison, and use. Model serving platforms facilitate online model execution.

A repository or serving platform may be provided as a hosted service, in which case models are deployed and made available to users via the Internet, or self-service, requiring users to operate the system locally and manage the deployment of models across their own infrastructure.

While many learning systems provide other capabilities (e.g., interactive computing interfaces via Jupyter notebooks [25]), we limit our survey to repository and serving capabilities.

\section{A. Model Repositories}

Model repositories catalog and aggregate models, often by domain, storing trained and untrained models with associated metadata to enable discovery and citation. Metadata may be user-defined and/or standardized by using common publication schemas (e.g., author, creation date, description, etc.) and ML-specific schemas. ML-specific metadata include modelspecific metadata (e.g., algorithm, software version, network 
architecture), development provenance (e.g., versions, contributors), training metadata (e.g., datasets and parametrization used for training), and performance metadata (e.g., accuracy when applied to benchmark datasets). Model repositories may provide the ability to associate a persistent identifier (e.g., DOI) and citation information such that creators may receive credit for their efforts.

Table II summarizes four representative model repositories plus DLHub along the following dimensions; we describe each repository in more detail below.

- Publication and curation: Can models be contributed by users and is any curation process applied.

- Domain: Whether the repository is designed for a single domain (e.g., bioinformatics) or for many domains.

- Model types: What types of ML models can be registered in the repository (e.g., any model type, TensorFlow)

- Data integration: Whether data (e.g., training/test datasets) and configuration (e.g., hyperparameters) can be included with the published model.

- Model metadata: Whether the repository supports publication, model-specific, model building, and/or invocation metadata.

- Search capabilities: What search mechanisms are provided to allow users to find and compare models.

- Model versioning: Whether the repository facilitates versioning and updates to published models.

- Export: Does the repository allow models to be exported and if so in what format.

1) ModelHub [6] is a deep learning model lifecycle management system focused on managing the data artifacts generated during the deep learning lifecycle, such as parameters and $\operatorname{logs}$, and understanding the behavior of the generated models. Using a Git-like command line interface, users initialize repositories to capture model information and record the files created during the creation process. Users then exchange a custom-built model versioning repository, called DLV, through the hosted service to enable publication and discovery. ModelHub is underpinned by Git, inheriting versioning capabilities, support for arbitrary datasets, scripts, and features, and accommodates models regardless of domain. A custom SQLlike query language, called DQL, allows ModelHub users to search across repositories by characteristics such as authors, network architecture, and hyper-parameters.

2) Caffe Model Zoo [26] is a community-driven effort to publish and share Caffe [7] models. Users contribute models via Dropbox or Github Gists. The Model Zoo provides a standard format for packaging, describing, and sharing Caffe models. It also provides tools to enable users to upload models and download trained binaries. The Model Zoo operates a community-edited Wiki page to describe each of the published models, aggregating information regarding manuscripts, citation, and usage documentation in an unstructured format. The project encourages open sharing of models, trained weights, datasets, and code through Github. The Model Zoo provides guidelines on how to contribute models and what metadata should be included in the accompanying readme.md file without enforcing a specific schema. Users typically include citation information (e.g., BibTeX references to papers), links to the project page, a Github address for the model's code, and in some cases, a link to haystack.ai where the model can be tested.

3) ModelHub.ai [27] is a service to crowdsource and aggregate deep learning models related to medical applications. ModelHub.ai has a Web interface that lets users review published models, experiment with example inputs, and even test them online using custom inputs. The service provides detailed documentation and libraries to package models into a supported Docker format. Once packaged, users can add the model and any associated metadata to the ModelHub GitHub repository and submit a pull-request. The contributed model is curated and added to the catalog. The ModelHub.ai project provides both a Flask and Python API to interact with Dockerized models, which can be retrieved by either downloading the Docker image or cloning the GitHub repository.

4) Kipoi [4] is a repository of trained models for genomics that includes more than 2000 models of 21 different types. It provides a command line interface (CLI) for publishing and accessing models. On publication, the CLI queries the user for descriptive metadata and generates a configuration file containing the metadata needed to discover and run the model. Users can then publish their models by submitting a pull-request to the Kipoi GitHub repository. Models can be listed and retrieved through the API and then invoked locally.

\section{B. Model Serving}

ML model serving platforms provide on-demand model inference. Existing model serving platforms vary in both their goals and capabilities: for example, some focus on serving a specific type of model with extremely low latency, while others prioritize ease of use and simple inference interfaces. We have identified the following important dimensions to capture the differences between model serving platforms. Table II summarizes popular model serving platforms plus DLHub along these dimensions.

- Service model: Whether the platform is offered as a hosted service or requires self-service deployment.

- Model types: What languages and types are supported (e.g., TensorFlow, Scikit-learn, R, Python, etc.)

- Input types: The range of input types supported by the system. (e.g., structured, files, or primitive types)

- Training capabilities: Whether the system supports training.

- Transformations: Whether pre-/post-processing steps can be deployed.

- Workflows: Support for workflows between models and transformation codes.

- Invocation interface: Methods of interaction with the models.

- Execution environment: Where models are deployed (e.g., cloud, Kubernetes, Docker). 
TABLE I: Model repositories compared and contrasted. BYO = bring your own.

\begin{tabular}{|l|c|c|c|c|c|}
\hline & ModelHub & Caffe Zoo & ModelHub.ai & Kipoi & DLHub \\
\hline Publication method & BYO & BYO & Curated & Curated & BYO \\
\hline Domain(s) supported & General & General & Medical & Genomics & General \\
\hline Datasets included & Yes & Yes & No & No & Yes \\
\hline Metadata type & Ad hoc & Ad hoc & Ad hoc & Structured & Structured \\
\hline Search capabilitiees & SQL & None & Web GUI & Web GUI & Elasticsearch \\
\hline Identifiers supported & No & BYO & No & BYO & BYO \\
\hline Versioning supported & Yes & No & No & Yes & Yes \\
\hline Export method & Git & Git & Git/Docker & Git/Docker & Docker \\
\hline
\end{tabular}

TABLE II: Serving systems compared and contrasted. K8s = Kubernetes.

\begin{tabular}{|l|c|c|c|c|c|}
\hline & PennAI & TF Serving & Clipper & SageMaker & DLHub \\
\hline Service model & Hosted & Self-service & Self-service & Hosted & Hosted \\
\hline Model types & Limited & TF Servables & General & General & General \\
\hline Input types supported & Unknown & Primitives, Files & Primitives & Structured, Files & Structured, Files \\
\hline Training supported & Yes & No & No & Yes & No \\
\hline Transformations & No & Yes & No & No & Yes \\
\hline Workflows & No & No & No & No & Yes \\
\hline Invocation interface & Web GUI & gRPC, REST & gRPC, REST & gRPC, REST & API, REST \\
\hline Execution environment & Cloud & Docker, K8s, Cloud & Docker, K8s & Cloud, Docker & K8s, Docker, Singularity, Cloud \\
\hline
\end{tabular}

1) PennAI [28] provides model serving capabilities for biomedical and health data. The platform allows users to apply six ML algorithms, including regressions, decision trees, SVMs, and random forests to their datasets, and to perform supervised classifications. The PennAI website provides a user-friendly interface for selecting, training, and applying algorithms to data. The platform also exposes a controller for job launching and result tracking, result visualization tools, and a graph database to store results. PennAI does not support user-provided models, but does provide an intuitive mechanism to train classification tools and simplify the integration of ML into scientific processes.

2) TensorFlow Serving [29] is the most well-known model serving solution and is used extensively in production environments. TensorFlow Serving provides high performance serving via gRPC and REST APIs and is capable of simultaneously serving many models, with many versions, at scale. TensorFlow Serving provides the lowest latency serving of any of the surveyed platforms. It serves trained TensorFlow models using the standard tensorflow_model_server, which is built in C++. Although TensorFlow Serving does support a range of model types - those that can be exported into TensorFlow servables-it is limited in terms of its support for custom transformation codes and does not support the creation of pipelines between servables. TensorFlow Serving is also self-service, requiring users to deploy and operate TensorFlow Serving on local (or cloud) infrastructure in order to deposit models and perform inferences. TensorFlow also provides model repository capabilities through a library of reusable ML modules, called TensorFlow Hub.

3) SageMaker [3] is a ML serving platform provided by Amazon Web Services that supports both the training of models and the deployment of trained models as Docker containers for serving. It helps users to handle large data efficiently by providing ML algorithms that are optimized for distributed environments. SageMaker APIs allow users to deploy a variety of ML models and integrate their own algorithms. In addition, trained models can be exported as Docker containers for local deployment.

4) Clipper [5] is a prediction serving system that focuses on low latency serving. It deploys models as Docker containers, which eases management complexity and allows each model to have its own dependencies wrapped in a self-contained environment. Clipper includes several optimizations to improve serving performance including data batching and memoization. Clipper also provides a model selection framework to improve prediction accuracy. However, because Clipper needs to dockerize the models on the manager node, it requires privileged access, which is not available on all execution environments (e.g., high performance computing clusters).

5) Kubeflow [30] is a collection of open source ML services that can be deployed to provide a fully-functional ML environment on a Kubernetes cluster. The system simplifies the deployment of various ML tools and services, including those to support model training, hyper-parameter tuning, and model serving. Kubeflow also integrates Jupyter Notebooks to provide a user-friendly interface to many of these services. Model serving in Kubeflow is achieved with TensorFlow Serving, therefore we have not included it in our summary table. However, using TensorFlow Serving provides Kubeflow with a low-latency model serving solution while adding additional capabilities (e.g., training) to the platform.

\section{DLHub ARChitecture AND IMPLEMENTATION}

DLHub is a learning system that provides model publishing and serving capabilities for scientific ML. DLHub's model repository supports user-driven publication, citation, discovery, and reuse of ML models from a wide range of domains. It offers rich search capabilities to enable discovery of, and access to, published models. DLHub automatically converts each published model into a "servable"-an executable DLHub container that implements a standard execution interface 
and comprises a complete model package that includes the trained model, model components (e.g., training weights, hyperparameters), and any dependencies (e.g., system or Python packages). DLHub can then "serve" the model by deploying and invoking one or more instances of the servable on execution site(s). DLHub provides high throughput and lowlatency model serving by dispatching tasks in parallel to the remote execution site(s). DLHub implements a flexible executor model in which several serving infrastructures, including TensorFlow Serving, SageMaker, and our generalpurpose Parsl-based execution platform, can be used to execute tasks. The DLHub architecture, shown in Fig. 2, comprises three core components: the Management Service, one or more Task Managers, and one or more Executors.

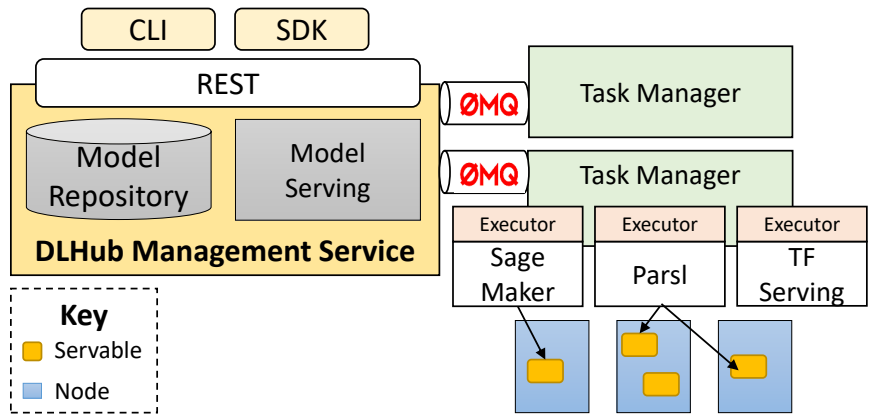

Fig. 2: DLHub architecture. User requests, submitted via REST, SDK, or CLI (upper left) can result in model publication in the Repository or the dispatch of serving requests to servables deployed on any computing resources with a Task Manager interface and appropriate executor(s) (lower right).

\section{A. Management Service}

DLHub's Management Service is the user-facing interface to DLHub. It enables users to publish models, query available models, execute tasks (e.g., inference), construct pipelines, and monitor the status of tasks. The Management Service includes advanced functionality to build models, optimize task performance, route workloads to suitable executors, batch tasks, and cache results.

Model Repository: A primary function of the Management Service is to implement model repository functionality to support the publication and discovery of models. DLHub defines a model publication schema that is used to describe all published models. The schema includes standard publication metadata (e.g., creator, date, name, description) as well as MLspecific metadata such as model type (e.g., Keras, TensorFlow) and input and output data types. These metadata are registered in a Globus Search [31] index that can be queried by users to discover appropriate models.

Model discovery: DLHub's search interface supports finegrained, access-controlled queries over model metadata. When a new model is published in DLHub, its metadata are indexed in Globus Search. It can the be queried using free text queries, partial matching, range queries, faceted search, and more.
Servables: In order to provide a common model execution interface irrespective of model type, DLHub converts all published models into executable servables. Thus, DLHub requires that users upload all model components as well as descriptive metadata for building the servable. As model components can be large, model components can be uploaded to an AWS S3 bucket or a Globus endpoint. Once a model is published, the Management Service downloads the components and builds the servable in a DLHub-compatible format. It combines DLHub-specific dependencies with usersupplied model dependencies into a Dockerfile. It then uses the Dockerfile to create a Docker container with the uploaded model components and all required dependencies. Finally, it uploads the container to the DLHub model repository, and registers the container location in the Globus Search index alongside the user-supplied descriptive metadata.

Model serving: The Management Service also coordinates the execution of tasks on remote resources. It uses a $\mathrm{Ze}$ roMQ [32] queue to send tasks to registered Task Managers for execution. The queue provides a reliable messaging model that ensures tasks are received and executed. When a user invokes a model (or executes another task), with some input data, the Management Service packages up the request and posts it to a ZeroMQ queue. Registered Task Managers can retrieve waiting tasks from the queue, unpackage the request, execute the task, and return the results via the same queue. DLHub supports both synchronous and asynchronous task execution. In asynchronous mode, the Management Service returns a unique task UUID that can be used subsequently to monitor the status of the task and retrieve its result.

\section{B. Task Managers}

Any compute resource on which DLHub is to execute tasks must be preconfigured with DLHub Task Manager software. The Task Manager is responsible for monitoring the DLHub task queue(s) and then executing waiting tasks. The Task Manager is responsible for deploying servables using one of the supported executors and then routing tasks to appropriate servables. When a Task Manager is first deployed it registers itself with the Management Service and specifies which executors and DLHub servables it can launch. The Task Manager can be deployed in Docker environments, Kubernetes clusters, and HPC resources via Singularity.

\section{Executors}

DLHub aims to provide efficient model execution for a wide range of model types. To achieve this goal it implements an arbitrary executor model that currently supports three serving systems: TensorFlow Serving, SageMaker, and a general-purpose Parsl executor. The first two executors work only with containers deployed to Kubernetes, while the Parsl executor can support Kubernetes and many other common HPC schedulers and clouds.

When invoking a given servable the Task Manager determines to which executor to send the task. Inference tasks are sent to the appropriate serving executor, other types 
of tasks (e.g., pre/post processing) are sent to the general Parsl executor. Each executor is responsible for running the task, translating the results into a common DLHub executorindependent input format, and returning the results back to the Management Service via the Task Manager. While servables might be invoked through different executors, all requests are managed by the Management Service and benefit from the same batching, memoization, and workflow capabilities,

TensorFlow Serving executor: To invoke TensorFlow models, the Task Manager first deploys one or more TensorFlow Serving containers on Kubernetes. It then routes subsequent TensorFlow servable invocations to these containers. The executor uses Google's low-latency gRPC protocol for high performance model inference.

SageMaker executor: The SageMaker executor deploys one or more SageMaker containers on Kubernetes. The SageMaker container includes a Python Flask application that exposes an HTTP-based model inference interface. The Task Manager composes HTTP requests to the SageMaker interface to perform inference.

Parsl executor: The Parsl executor uses the Parsl [33] parallel scripting library's execution engine to execute tasks on arbitrary resources. This execution engine supports the execution of Python functions, arbitrary executables, and containerized applications on different platforms, via a modular execution interface that allows a client (in our case, the Task Manager) to specify the desired execution location and associated parameters, such as degree of parallelism.

Implementations of the Parsl execution engine on cluster, cloud, and supercomputer platforms, among others, use platform-specific mechanisms to dispatch tasks and their data, initiate task execution, monitor progress, and report on results. On a Kubernetes cluster, for example, the engine creates a Kubernetes Deployment consisting of $n$ pods for each servable that is to be executed, a number configurable in the Management Service. Parsl then deploys IPythonParallel (IPP) engines in each servable container and connects back to the Task Manager to retrieve servable execution requests. Parsl dispatches requests to the appropriate containers using IPP, load balancing them automatically across the available pods.

\section{Security}

DLHub implements a comprehensive security model that ensures that all operations are performed by authenticated and authorized users. To do so, we rely on Globus Auth-a flexible authentication and access management service that is designed to broker authentication and authorization decisions between users, identity providers, resource serves, and clients.

The DLHub Management Service is registered as a Globus Auth resource server [34] with associated scope for programmatic invocation. Users can authenticate with DLHub using one of hundreds of supported identity providers (e.g., campus, ORCID, Google). When authenticating, the Management Service first validates the user's identity, and then retrieves shortterm access tokens that allow it to obtain linked identities, profile information about the user, and to access/download data on their behalf. These capabilities allow DLHub to precomplete publication metadata using profile information and also to transfer model components and inputs from Globus endpoints seamlessly.

\section{E. DLHub interfaces}

DLHub offers a REST API, Command Line Interface (CLI), and a Python Software Development Kit (SDK) for publishing, managing, and invoking models. We also provide a user toolbox to assist with the creation of metadata that adhere to DLHub model schemas.

The DLHub toolbox supports programmatic construction of JSON documents that specify publication and model-specific metadata that complies with DLHub-required schemas. The resulting documents can then be uploaded to DLHub to publish the model. The toolbox also provides functionality to execute DLHub models locally. This functionality is useful for model development and testing.

The DLHub CLI provides an intuitive user interface to interact with DLHub. It provides a Git-like interface with commands for initializing a DLHub servable in a local directory, publishing the servable to DLHub, creating metadata using the toolbox, and invoking the published servable with input data. Supported commands include:

- init: Initialize a servable in the current working directory on the user's computer, creating a . dl hub directory and a metadata file for the servable.

- update: Modify a servable's published metadata.

- publish: Push a locally created servable (model and metadata) to DLHub.

- run: Invoke a published servable with appropriate input arguments.

- Is: List the servables that are being tracked on the user's computer.

The DLHub Python SDK supports programmatic interactions with DLHub. The SDK wraps DLHub's REST API, providing access to all model repository and serving functionality.

\section{Evaluation}

To evaluate DLHub we conducted experiments to explore its serving performance, the impacts of memoization and data batching, and Task Manager scalability. We also compared its serving infrastructure against those of TensorFlow Serving, Clipper, and SageMaker.

\section{A. Experimental Setup}

We use Argonne National Laboratory's PetrelKube, a 14node Kubernetes cluster, as a compute resource for these experiments. Each node has two E5-2670 CPUs, 128GB RAM, two 300GB hard drives in RAID 1, two 800GB Intel P3700 NVMe SSDs, and 40GbE network interconnection.

In our experiments, we deployed a single Task Manager on a co-located cluster, Cooley, within Argonne's Leadership Computing Facility. The average Internet Protocol round-triptime between the Task Manager and PetrelKube, where the servables are deployed, is $0.17 \mathrm{~ms}$. The Management Service 
was deployed on an Amazon EC2 instance and has an average round-trip-time to the Task Manager of $20.7 \mathrm{~ms}$. These overheads are consistent across our tests and are present regardless of executor or serving infrastructure used.

When reporting the end-to-end performance of servable invocations we use the following metrics.

- Inference time is captured at the servable and measures the time taken by the servable to run the component.

- Invocation time is captured at the Task Manager and measures elapsed time from when a request is made to the executor to when the result is received from the servable.

- Request time is captured at the Management Service and measures the time from receipt of the task request to receipt of its result from the Task Manager.

- Makespan is the completion time of serving all the submitted requests.

We use six servables to compare the different serving infrastructures. The first is a baseline "noop" task that returns "hello world" when invoked. The second is Google's 22-layer Inception-v3 [35] model ("Inception"). Inception is trained on a large academic dataset for image recognition and classifies images into 1000 categories. Inception takes an image as input and outputs the five most likely categories.

The third is a multi-layer convolutional neural network trained on CIFAR-10 [36] ("CIFAR-10"). This common benchmark problem for image recognition takes a $32 \times 32$ pixel RGB image as input and classifies it in 10 categories.

The final three servables are part of a workflow used to predict the stability of a material given its elemental composition (e.g., $\mathrm{NaCl}$ ). The model is split into three servables: parsing a string with pymatgen [37] to extract the elemental composition ("matminer_util"), computing features from the element fractions by using Matminer [38] ("matminer_featurize"), and executing a scikit-learn random forest model to predict stability ("matminer_model"). The model was trained with the features of Ward et al. [39] and data from the Open Quantum Materials Database [40].

\section{B. Experiments}

To remove bias we disable DLHub memoization mechanisms and restrict data batch size to one in the following experiments, except where otherwise noted. Although both the Management Service and Task Manager are multi-threaded, we conduct all experiments sequentially, waiting for a response before submitting the next request.

1) Servable Performance. To study the performance of the DLHub components (i.e., servable, Management Service, and Task Manager) we measure request, invocation, and inference times when requests are issued via the Management Service. We evaluate six servables on PetrelKube, submitting 100 requests with fixed input data to each.

Fig. 3 shows the inference, invocation, and request times for each of the six servables. The difference between inference and invocation times reflects costs associated with the Task Manager while the difference between invocation and request times reflects costs associated with the Management
Service. In most cases, costs are around 10-20ms; as these include round-trip-time communication times, it seems that DLHub imposes only modest additional overhead. The higher overheads associated with Inception and CIFAR-10 are due to their need to transfer substantial input data.

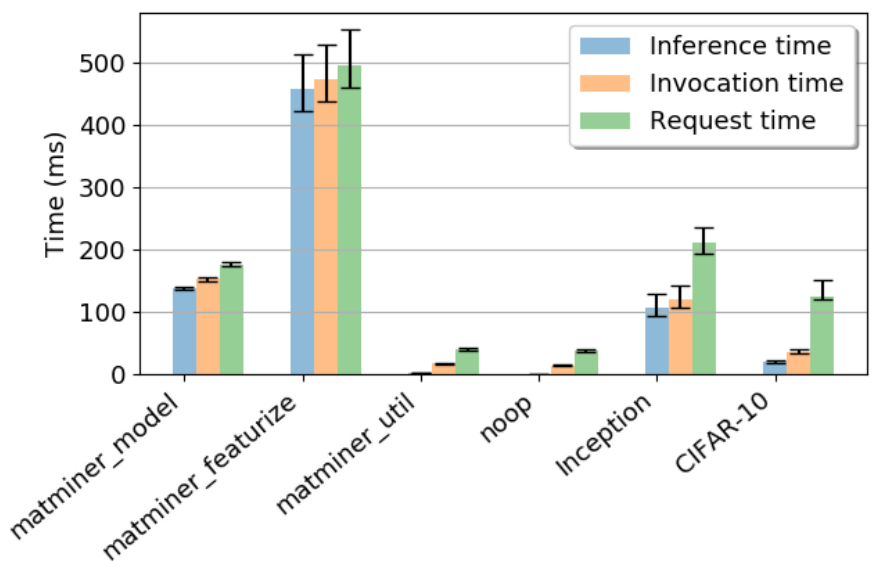

Fig. 3: Request, invocation, and inference times for six servables. Bars give median values and the error bars the 5th and 95th percentile values.

2) Memoization. DLHub's Parsl executor implements memoization [41], caching the inputs and outputs for each request and returning the recorded output for a new request if its inputs are in the cache. To investigate the effect of memoization on serving performance we submitted requests with the same input as in 8 -B1, with memoization variously enabled and disabled. Fig. 4 shows invocation and request times. Inference time is not shown in the figure as memoization removes the need to execute the inference task. We observe that memoization reduces invocation time by $95.3-99.8 \%$ and request time by $24.3-95.4 \%$.

3) Batching. DLHub support for batch queries is designed to improve overall throughput by amortizing system overheads over many requests. We studied how performance varies with batch size. First, we measured the time required to process numbers of requests in the range $[1,100]$ for three example servables, with and without batching. Due to space limitations, we show only invocation time in Fig. 5. We see that batching significantly reduces overall invocation time. Next, we measured invocation time for the same three servables with batching, as the number of requests scales far higher, to 10,000 . We see in Fig. 6 a roughly linear relationship between invocation time and number of requests. In future work, we intend to use such servable profiles to design adaptive batching algorithms that intelligently distribute serving requests to reduce latency.

4) Scalability. We performed throughput tests with the Parsl executor, with memoization disabled and a batch size of one. Fig. 7 shows observed Task Manager throughput for the Inception, CIFAR-10, and Matminer_featurize models as the number of deployed model replicas is increased. We find that behavior varies with model. For example, when serving Inception requests, throughput increases rapidly up to $\sim 15$ 

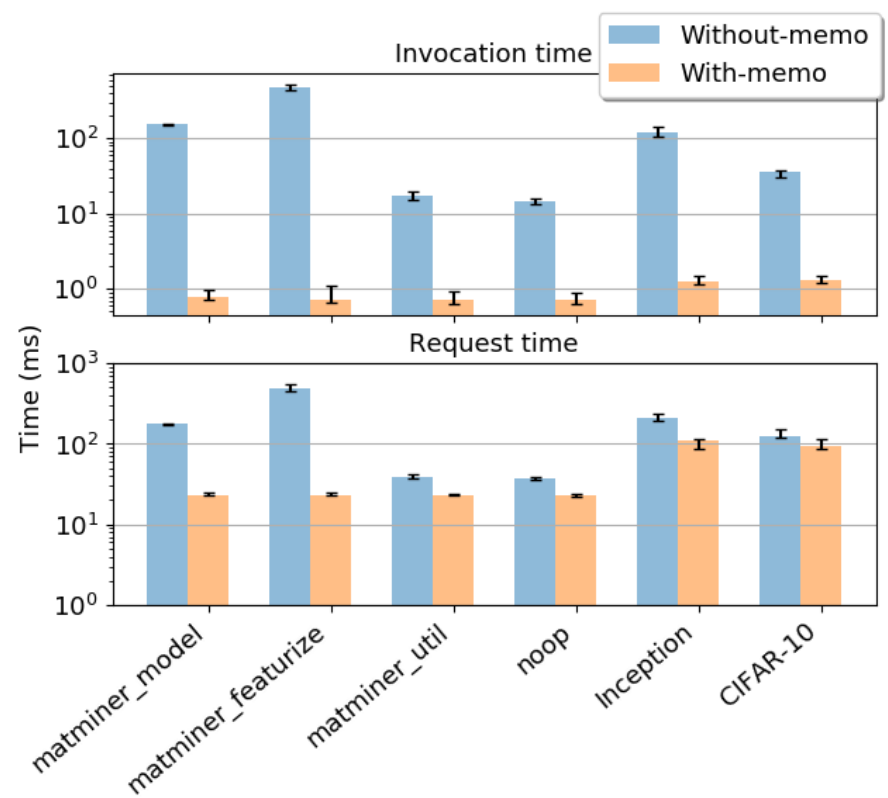

Fig. 4: Performance impact of memoization. Bars and error bars show median and 5th/95th percentiles, as in Fig. 3 .
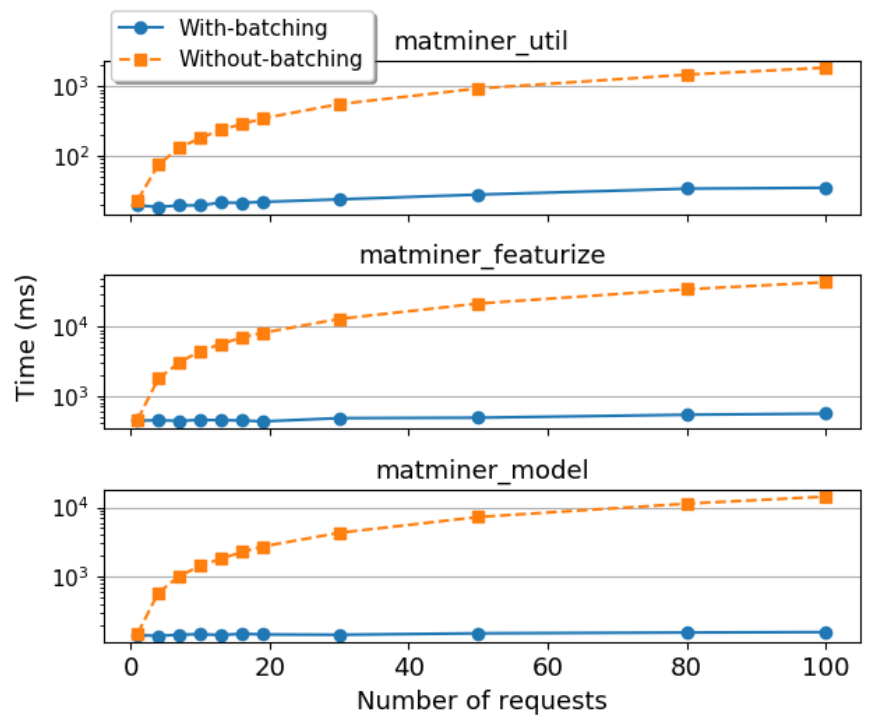

Fig. 5: Servable invocation time, with and without batching.

replicas, after which subsequent replicas have diminishing effect and executor throughput eventually saturates. As we expect, servables that execute for shorter periods benefit less from additional replicas, presumably because task dispatch activities eventually come to dominate execution time.

5) Serving Comparison. We used CIFAR-10 and Inception to compare the serving performance of TensorFlow Serving, SageMaker, Clipper, and DLHub. For TensorFlow Serving, we export the trained models and use the standard tensorflow_model_server. For SageMaker, we use the SageMaker service to create the models, which we then deploy in DLHub. For Clipper, we use its Kubernetes container man-

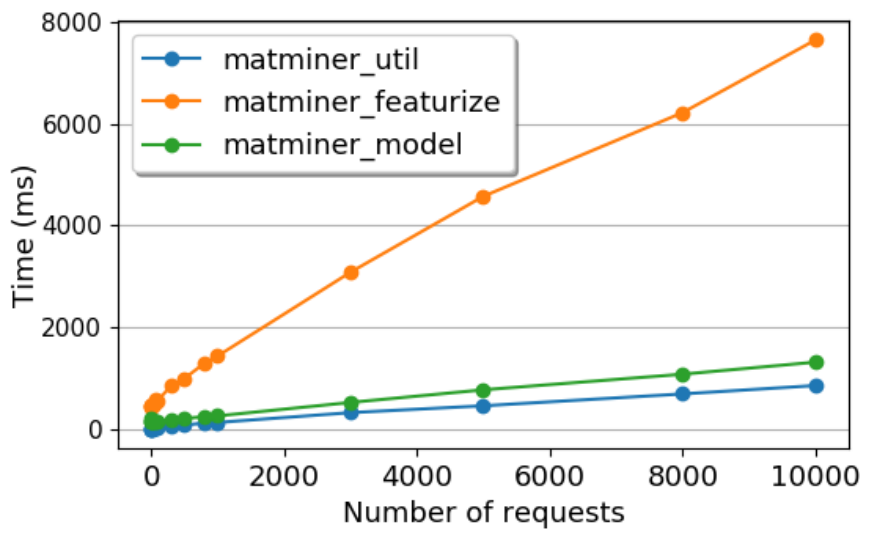

Fig. 6: Invocation time vs. number of requests, with batching.

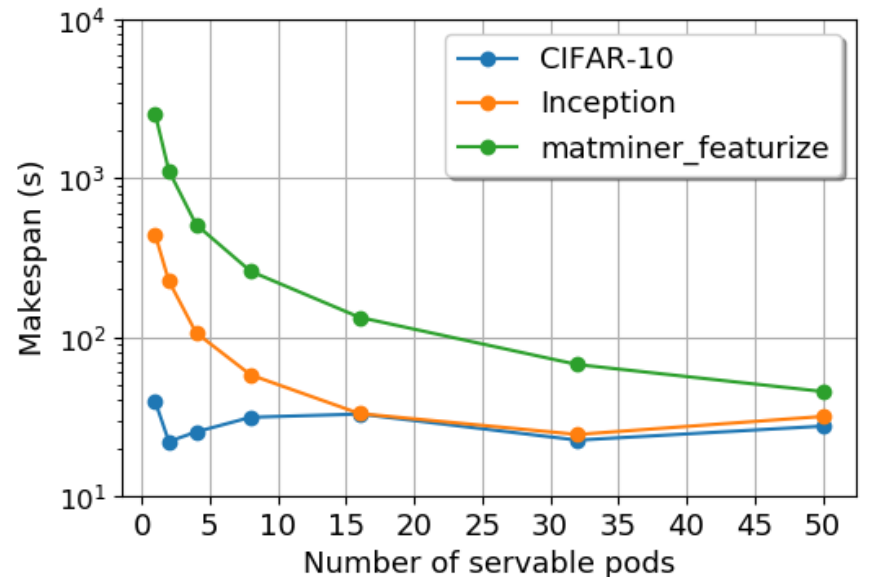

Fig. 7: The time required for three different models to process 5,000 inferences for varying numbers of replicas.

ager to deploy it on PetrelKube and register the CIFAR-10 and Inception models with it. For DLHub, we serve the two models with the Parsl executor. We evaluated serving performance by submitting requests to the Management Service and having the Task Manager route requests to each platform. We report the average time from 100 requests for each model and platform.

TensorFlow Serving provides two model serving APIs: REST and gRPC. SageMaker also supports serving TensorFlow models through TensorFlow Serving or its native Flask framework. In our experiments, we explore all possible APIs and frameworks, i.e., TFServing-REST, TFServinggRPC, SageMaker-TFServing-REST, SageMaker-TFServinggRPC, SageMaker-Flask. In addition, as DLHub and Clipper both support memoization, we evaluate those two systems with and without memoization.

Figure 8 shows the invocation times and request times of CIFAR-10 and Inception using each serving system. We see that the servables invoked through the TensorFlow Serving framework (i.e., TFServing-gRPC, TFServing-REST and SageMaker-TFServing) outperform those using other serving systems (SageMaker-Flask and DLHub) in terms of both invocation time and request time. This is because the core 
tensorflow_model_server, implemented in $\mathrm{C}++$, outperforms Python-based systems. DLHub's performance is comparable to the other Python-based serving infrastructures. In addition, gRPC leads to slightly better performance than REST due to the overhead of the HTTP protocol. With memoization enabled, DLHub provides extremely low invocation times (1ms) - much lower than other systems. This is because Parsl maintains a cache at the Task Manager, greatly reducing serving latency. Clipper, in contrast, maintains a cache at the query frontend that is deployed as a pod on the Kubernetes cluster (PetrelKube). Hence, cached responses still require the request to be transmitted to the query frontend, leading to additional overhead.

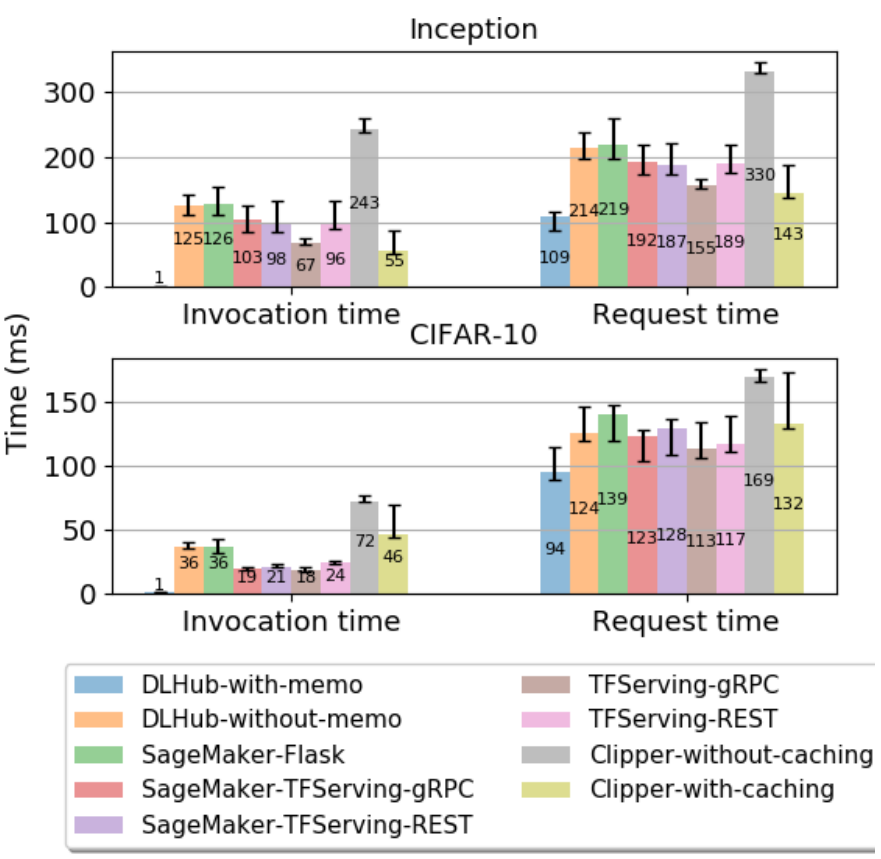

Fig. 8: Performance of different serving systems on the Inception and CIFAR-10 problems.

\section{USE CASES}

To illustrate the value of DLHub we briefly outline four use cases that exemplify early adoption of the system.

\section{A. Publication of Cancer Research Models}

The Cancer Distributed Learning Environment (CANDLE) project [42] leverages leadership scale computing resources to address problems relevant to cancer research at different biological scales, specifically problems at the molecular, cellular, and population scales. CANDLE uses DLHub to securely share and serve a set of deep learning models and benchmarks using cellular level data to predict drug response based on molecular features of tumor cells and drug descriptors. As the models are still in development, they require substantial testing and verification by a subset of selected users prior to their general release. DLHub supports this use case by supporting model sharing and discovery with fine grain access control.
Thus, only permitted users can discover and invoke the models through the platform. Once models are determined suitable for general release, the access control on the model can be updated within DLHub to make them publicly available.

\section{B. Enriching Materials Datasets}

The Materials Data Facility [23] (MDF) is a set of data services developed to enable data publication and data discovery in the materials science community. MDF allows researchers to distribute their data, which may be large or heterogeneous, and rapidly find, retrieve, and combine the contents of datasets indexed from across the community. MDF leverages several models published in DLHub to add value to datasets as they are ingested. When a new dataset is registered with MDF, automated workflows [31] are applied to trigger the invocation of relevant models to analyze the dataset and generate additional metadata. The selection of appropriate models is possible due to the descriptive schemas used in both MDF and DLHub. MDF extracts and associates fine-grained type information with each dataset which are closely aligned with the applicable input types described for each DLHub model.

\section{Processing Tomographic Neuroanatomy Data}

DLHub is used by a research group using X-ray microtomgraphy at the Advanced Photon Source to rapidly characterize the neuroanatomical structure of large $\left(\mathrm{cm}^{3}\right)$ unsectioned brain volumes in order to study brain aging and disease [43]. During the image reconstruction process, it is important to identify the center of the imaged sample from the hundreds of slices with varying quality. A DLHub model is used to aid in the identification of the highest quality slice to be used for tomographic reconstruction. Once reconstructed, the resulting images are further processed with segmentation models to characterize cells. These results can then be used to visualize and analyze the composition of the brain tissue. DLHub hosts both the center-finding and segmentation models, enabling near real-time automated application of the center finding models during the reconstruction process as well as facilitating batch-style segmentation post-processing of images.

\section{Predicting Formation Enthalpy}

DLHub makes it easy to link models plus pre- and postprocessing transformations in pipelines to simplify the user experience. For example, a pipeline for predicting formation enthalpy from a material composition (e.g., $\mathrm{SiO}_{2}$ ) can be organized into three steps: 1) conversion of material composition text into a pymatgen [37] object; 2) creation of a set of features, via matminer [38], using the pymatgen object as input; and 3) prediction of formation enthalpy using the matminer features as input. Once the pipeline is defined, the end user sees a simplified interface that allows them to input a material composition and receive a formation enthalpy. This and other more complex pipelines are defined as a series of modularized DLHub servables within the execution environment. Defining these steps as a pipeline means data are automatically passed between each servable in the pipeline, meaning the entire 
execution is performed server-side, drastically lowering both the latency and user burden to analyze inputs.

\section{CONCLUSION}

The increasing reliance on ML in science necessitates the development of new learning systems to support the publication and serving of models. To address these requirements we have developed DLHub, a unique model repository and serving system that provides standardized, self-service model publication, discovery, and citation; scalable execution of inference tasks on arbitrary execution resources; integration with large-scale research storage systems; and support for flexible workflows. We showed that DLHub performs comparably with other serving platforms, and significantly better when its memoization and batching capabilities can be used, and can provide low latency inference.

In ongoing research, we are working to expand the set of models included in DLHub, simplify use by domain scientists, and study how DLHub capabilities are used in practice. We are investigating the utility of integrating multiple servables into single containers and optimization techniques for automated tuning of servable execution. We will also pursue the integration of scalable model training into DLHub workflows and work to further decrease model serving latencies.

\section{ACKNOWLEDGMENTS}

This work was supported in part by Laboratory Directed Research and Development (LDRD) funding from Argonne National Laboratory and the RAMSES project, both from the U.S. Department of Energy under Contract DE-AC0206CH11357. We thank Amazon Web Services for research credits and Argonne for computing resources.

\section{REFERENCES}

[1] P. Balaprakash et al., "AutoMOMML: Automatic multi-objective modeling with machine learning," in International Conference on High Performance Computing. Springer, 2016, pp. 219-239.

[2] "Google Cloud AutoML," https://cloud.google.com/automl/ Accessed October 14, 2018.

[3] "Amazon SageMaker," https://docs.aws.amazon.com/sagemaker/latest/ dg/whatis.html Accessed October 14, 2018.

[4] Z. Avsec et al., "Kipoi: Accelerating the community exchange and reuse of predictive models for genomics," bioRxiv, vol. 10.1101/375345, 2018

[5] D. Crankshaw et al., "Clipper: A low-latency online prediction serving system," in 14th USENIX Symposium on Networked Systems Design and Implementation (NSDI), 2017, pp. 613-627.

[6] H. Miao et al., "Towards unified data and lifecycle management for deep learning," in 33rd Intl Conf. on Data Engineering. IEEE, 2017, pp. 571-582.

[7] Y. Jia et al., "Caffe: Convolutional architecture for fast feature embedding," in 22nd ACM Intl. Conf. on Multimedia, 2014, pp. 675-678.

[8] M. Abadi et al., "TensorFlow: A system for large-scale machine learning," in $O S D I$, vol. 16, 2016, pp. 265-283.

[9] F. Chollet, Deep Learning with Python. Manning Publications, 2017.

[10] F. Pedregosa et al., "Scikit-learn: Machine learning in Python," Journal of Machine Learning Research, vol. 12, no. Oct, pp. 2825-2830, 2011.

[11] K. Chard et al., "Globus: Recent enhancements and future plans," in XSEDE16 Conference on Diversity, Big Data, and Science at Scale. ACM, 2016, p. 27.

[12] C. Olston et al., "TensorFlow-Serving: Flexible, high-performance ML serving," in 31st Conf. on Neural Information Processing Systems, 2017.

[13] O. E. Gundersen et al., "State of the art: Reproducibility in artificial intelligence," in 30th AAAI Conf. on Artificial Intelligence, 2017.
[14] E. Gossett et al., "AFLOW-ML: A RESTful API for machine-learning predictions of materials properties," Computational Materials Science, vol. 152, pp. 134-145, 2018.

[15] Q. Zhang et al., "OCPMDM: Online computation platform for materials data mining," Chemometrics and Intelligent Laboratory Systems, vol. 177, no. November 2017, pp. 26-34, 2018.

[16] A. Agrawal et al., "An online tool for predicting fatigue strength of steel alloys based on ensemble data mining," International Journal of Fatigue, vol. 113, pp. 389-400, 2018.

[17] M. Baker, "1,500 scientists lift the lid on reproducibility," Nature, vol. 533, pp. 452-454, 2016.

[18] A. Morin et al., "Shining light into black boxes," Science, vol. 336, no. 6078, pp. 159-160, 2012.

[19] A. Brinckman et al., "Computing environments for reproducibility: Capturing the "Whole Tale"," Future Generation Computer Sys., 2018.

[20] V. Stodden et al., "Enhancing reproducibility for computational methods," Science, vol. 354, no. 6317, pp. 1240-1241, 2016.

[21] J. Towns et al., "XSEDE: Accelerating scientific discovery," Computing in Science \& Engineering, vol. 16, no. 5, pp. 62-74, 2014.

[22] R. Pordes et al., "The Open Science Grid," in Journal of Physics: Conference Series, vol. 78, no. 1. IOP Publishing, 2007, p. 012057.

[23] B. Blaiszik et al., "The Materials Data Facility: Data services to advance materials science research," JOM, vol. 68, no. 8, pp. 2045-2052, 2016.

[24] A. Jain et al., "The Materials Project: A materials genome approach to accelerating materials innovation," APL Materials, vol. 1, no. 1, p. 011002, 2013

[25] T. Kluyver et al., "Jupyter Notebooks-a publishing format for reproducible computational workflows." in ELPUB, 2016, pp. 87-90.

[26] "Caffe Model Zoo," http://caffe.berkeleyvision.org/model_zoo.html Accessed October 14, 2018

[27] "ModelHub," http://modelhub.ai/ Accessed October 14, 2018.

[28] R. S. Olson et al., "A system for accessible artificial intelligence," in Genetic Programming Theory and Practice XV. Springer, 2018, pp. 121-134.

[29] C. Olston et al., "Tensorflow-Serving: Flexible, high-performance ML serving," arXiv preprint arXiv:1712.06139, 2017.

[30] "Kubeflow," https://www.kubeflow.org/ Accessed April 1, 2018.

[31] R. Ananthakrishnan et al., "Globus platform services for data publication," in Practice and Experience on Advanced Research Computing. ACM, 2018, pp. 14:1-14:7.

[32] P. Hintjens, ZeroMQ: Messaging for Many Applications. O'Reilly Media, Inc., 2013.

[33] Y. Babuji et al., "Parsl: Scalable parallel scripting in Python," in 10th International Workshop on Science Gateways, 2018.

[34] R. Anathankrishnan et al., "Globus Auth: A research identity and access management platform," in 16th Intl Conf. on e-Science, 2016.

[35] C. Szegedy et al., "Rethinking the Inception architecture for computer vision," in IEEE Conference on Computer Vision and Pattern Recognition, 2016, pp. 2818-2826.

[36] A. Krizhevsky, "Learning multiple layers of features from tiny images," Citeseer, Tech. Rep., 2009.

[37] S. P. Ong et al., "Python Materials Genomics (pymatgen): A robust, open-source Python library for materials analysis," Computational Materials Science, vol. 68, pp. 314-319, 2013.

[38] L. Ward et al., "Matminer: An open source toolkit for materials data mining," Computational Materials Science, vol. 152, pp. 60-69, 2018.

[39] _ - "A general-purpose machine learning framework for predicting properties of inorganic materials," npj Computational Materials, vol. 2, p. $16028,2016$.

[40] S. Kirklin et al., "The Open Quantum Materials Database (OQMD): Assessing the accuracy of DFT formation energies," npj Computational Materials, vol. 1, p. 15010, 2015.

[41] D. Michie, “"memo' functions and machine learning," Nature, vol. 218, no. 5136, p. $19,1968$.

[42] J. M. Wozniak et al., "CANDLE/Supervisor: A workflow framework for machine learning applied to cancer research," in Computational Approaches for Cancer Workshop, 2017.

[43] R. Chard et al., "High-throughput neuroanatomy and trigger-action programming: A case study in research automation," in 1st International Workshop on Autonomous Infrastructure for Science. ACM, 2018, p. 1. 